\title{
Die drei Ortssprachen Estlands in Edzard Schapers Roman Der Henker und in seiner estnischen Übersetzung ${ }^{1}$
}

\author{
MARIN JÄNES \\ MARIS SAAGPAKK
}

\begin{abstract}
The Three Local Languages of Estonia in Edzard Schaper's novel The Executioner and in its Estonian Translation. This article analyses the reflection of everyday multilingualism in Edzard Schaper's novel Der Henker (The Executioner, 1940) and its translation into Estonian by Katrin Kaugver (Timukas, 2002). The novel deals with the 1905 revolution in the current Estonian territory, which was at that time a province of the Russian Empire. The novel was written shortly before the outbreak of World War II and translated into Estonian 60 years later after the end of the Soviet era. The complexity and the fluctuation of the contextual elements between the storyline of the novel, the time of its writing and the time of the translation make the novel a rewarding object of research into settings of multilingualism in everyday life. The article focuses on the manifest and latent forms of multilingualism, on the functions of the local languages, as well as on the question whether it helps to analyse language use in real life situations. It also looks at how local multilingualism, dominated by three local languages - German, Russian and Estonian has been translated from one local language (German) into another local language (Estonian). The examples chosen in the article highlight some regularities in the use of the local and other languages, and offer a cultural-historical and socio-political interpretation of the use of multilingualism.
\end{abstract}

Keywords: literary multilingualism; Estonian; German; Russian; Baltic German; Edzard Schaper

1 Die Arbeit an diesem Artikel wurde unterstützt durch den Estnischen Wissenschaftsfonds, Grant PRG1206 („Translation in History, Estonia 1850-2010: Texts, Agents, Institutions and Practices"). 
JÄNES, SAAGPAKK

\section{Einleitung}

Die ethnisch gemischte Bevölkerung, Kontakte zu benachbarten Kulturen und die zeitgleiche oder sukzessive Zugehörigkeit der Gebiete zu verschiedenen Mächten formten aus dem Baltikum ${ }^{2}$ eine mehrsprachige Region. Neben den finnougrischen und baltischen Sprachen sind im Baltikum im Laufe der Geschichte viele Sprachen verwendet worden, von denen in früheren Zeiten besonders Latein und Niederdeutsch und später Deutsch und Russisch eine wichtige Rolle spielten. Da die Verwendung von Sprachen stets von sozialen Hierarchien und politischen Vorgaben abhängt, sind Erkenntnisse über die historische Mehrsprachigkeit für die Deutung unterschiedlicher historischer Prozesse interdisziplinär relevant. Auch Literatur kann hierbei als eine Quelle herangezogen werden. Ausgehend von der Feststellung des amerikanischen Mehrsprachigkeitsforschers Lawrence Alan Rosenwald - „literature can teach us something about linguistic history" (2008: xi) - wird im vorliegenden Beitrag der Verwendung der Sprachen anhand der Literatur nachgegangen.

Im Beitrag wird die Widerspiegelung der sprachlichen Vielfalt auf dem Territorium des heutigen Estlands im bekanntesten Werk von Edzard Schaper (1908 Ostrów [Ostrowo] - 1984 Bern) Der Henker ${ }^{3}$ (1940) und dessen estnischer Übersetzung von Katrin Kaugver Timukas ${ }^{4}$ (2002) analysiert. Der Roman, der sich mit der Revolution von 1905 beschäftigt, wurde am Vorabend des zweiten Weltkrieges geschrieben und 60 Jahre später übersetzt. Diese zeitlichen Sprünge sowie das jeweils unterschiedliche politische, soziale und kulturelle Umfeld während der Handlung, des Schreibens und des Übersetzens machen den Roman zu einem vielsprechenden Forschungsobjekt, das es ermöglicht, mehr über die jeweilige Sprachsituation zu erfahren.

Obwohl der Roman bereits in vielen Rezensionen und literaturwissenschaftlichen Beiträgen analysiert worden ist (z. B. Adson 1958; Lepik 1959; Neuschäffer 1982; Otto-Sprunck 1994; Illiste 1998; Lukas 1999; Undusk 1999; Arens 2009; Saagpakk 2014), hat der Aspekt der Mehrsprachigkeit bisher keine Beachtung gefunden, so wie der Einfluss der kolonial- und kontaktbeladenen sprachlichen Situation des Baltikums auf die Literatur generell nur am Rande erforscht worden ist (vgl. Wilpert 2005: 16).

2 Baltikum wird im Beitrag im Sinne des historischen Gebrauchs verwendet - vor dem ersten Weltkrieg wurden darunter die baltischen Provinzen des russischen Kaiserreichs (Est-, Liv- und Kurland) verstanden, was etwa den heutigen Staaten Est- und Lettland entspricht.

3 Erschienen im Insel-Verlag, später unter dem Titel Sie mähten gewappnet die Saaten.

4 Erschienen in der Buchreihe Livländische Klassik, eine Neuausgabe davon 2009 in der Reihe Estnische Geschichte. 
Die 1930-1940, während der in Estland verbrachten Jahre Schapers geleistete Vorarbeit zum Roman, wozu die Sichtung der Archivmaterialien, Zeitungen sowie der Protokolle von Behörden des zaristischen Russlands (Schaper im Interview mit Ex-., 1940: 10) gehörte, führte dazu, dass im Roman nicht nur die historischen Ereignisse, sondern laut Zeitzeugen auch die damaligen Umstände und Lebenslagen getreu weitergegeben werden. ${ }^{5}$ Geschrieben hauptsächlich auf Deutsch, kommen im Roman besonders die der konkreten Sprachsituation inhärenten Sprachen wie Estnisch und Russisch vor und sprachbezogene bzw. sprachpolitische Überlegungen bilden einen wichtigen Teil der Geschichte.

Im Beitrag wird gezeigt, in welchen manifesten und latenten Formen die Mehrsprachigkeit im Roman weitergegeben wird, welche Funktionen die drei lokalen Sprachen haben und welche Vermutungen daraus für tatsächliche Sprachsituationen gezogen werden können. Ebenso wird analysiert, wie die sprachliche Vielfalt, die von den drei Ortsprachen - Deutsch, Russisch, Estnisch - dominiert war, von einer der Ortssprachen (Deutsch) im Roman in die andere (Estnisch) der Übersetzung vermittelt wird und welche Verschiebungen dabei stattfinden. Da das Forschungsfeld der historischen Mehrsprachigkeit generell in einer Situation der „bad data“ arbeiten muss (Rutten, etc. 2017: 4), ist die literarische Darstellung der Mehrsprachigkeit trotz ihrer Literarizität und der daraus resultierenden Beschränktheit eine wertvolle Quelle. Anhand der Beispiele aus dem Roman und seiner Übersetzung wird auf einige Regelmäßigkeiten hingewiesen und eine kulturgeschichtliche und sozialpolitische Interpretation geboten.

\section{Der Henker und Timukas - der kontextuelle Rahmen des Romans und der Übersetzung}

Die Revolution von 1905, die im Baltikum neben der damals herrschenden russischen Kaisermacht gegen die deutschbaltische Oberschicht als lokale Machthaber gerichtet war, brachte eine historische Wende mit sich, da sie die Widersprüche zwischen verschiedenen politischen, sozialen und kulturellen Gruppen ans Tageslicht brachte. Diese stehen auch im Mittelpunkt des Romans Der Henker, in dem die Beziehungen der Deutschbalten und der Esten im russischen Kaiserreich während der Revolutionszeit dargestellt werden. Der historische Ausgangspunkt des Romans ist die Plünderung und Zerstörung

5 Z.B. Adson (1958: 59) sagt, dass Schaper als „Erster in der ausländischen Literatur ein so gründliches Bild von den damaligen sozialen, politischen und nationalen Umständen und Bestrebungen in unserem Land samt einer Gesamtübersicht unserer Geschichte gegeben hat." 
JÄNES, SAAGPAKK

von Gutshäusern während der Revolution im Dezember 1905, in deren Folge die zaristischen Straftruppen, unterstützt von deutschbaltischen Gutsherren, hunderte Menschen in Estland töteten, verhafteten und in die Verbannung schickten. Schaper stellt seine Hauptfigur Graf von Ovelacker, den Offizier deutschbaltischer Herkunft in Diensten der Russischen Armee, an die Spitze einer solchen Straftruppe, der im fiktiven Ort Drostenholm zuerst mit vier Offizieren über das Volk Gericht hält, danach aber den Dienst quittiert und als Erbe des ermordeten Gutsherrn in den selben Ort zurückkehrt. Spannung wird zwischen Ovelacker und dem estnischen Bauern Koiri Jaan aufgebaut. Koiri verliert in der Vergeltungsaktion gegen die Aufständischen zwei Söhne und sein dritter Sohn wird nach Sibirien verbannt. Im Kampf des Vaters um seine Befreiung und gegen den ,Henker 'Ovelacker kristallisiert sich das geschehene Unrecht. Die Geschichte endet mit gegenseitiger Vergebung der deutschbaltischen und estnischen Hauptfiguren. Lukas (vgl. 2009: 569) nennt den Roman deswegen einen,Versöhnungsroman'. Gierlichs Einschätzung „Seine [Schapers] Helden sind solche des Gewissen " (2014: [5]) trifft auch auf Den Henker zu, da die historischen Ereignisse den Rahmen um Fragen der Verantwortung, Schuld und Erlösung bilden.

Die Schreibzeit des Werkes fiel ans Ende der 1930er Jahre in der Republik Estland, in der die Deutschbalten aus der Position der Oberschicht in die einer Minderheit gedrängt worden waren und die deutschbaltische Siedlungsgeschichte im Baltikum sich dem Ende zu nähern begann. 1940, als Der Henker erschien, fing in Estland die erste sowjetische Okkupation an, an die sich 1941 die deutsche Okkupation anknüpfte, um 1944 durch die zweite sowjetische Okkupation ersetzt zu werden, die bis zur Wiederherstellung des selbstständigen Staates 1991 dauerte.

Schaper gehörte zu den Autoren, deren Werke während der Sowjetzeit nicht übersetzt und publiziert werden durften. ${ }^{6}$ So geschah es, dass die Übersetzung des Romans ins Estnische durch Katrin Kaugver mit dem Titel Timukas erst 2002 erschien. In den 60 Jahren zwischen dem Verfassen und Übersetzen des Werkes fanden in der Gesellschaft mehrere politische und kulturelle Umwälzungen statt, die ihren Einfluss auch auf die Übersetzung und ihre Rezeption ausübten.

Das turbulente politische, soziale und kulturelle Umfeld der Handlungszeit des Romans, die Schatten der Zeit des Schreibens und die Umstände seiner Übersetzungsgeschichte formen und gestalten die Mehrsprachigkeit im Text mit. Besonders präsent sind im Roman die drei, Ortssprachen ' und so wird im

6 In der exilestnischen Gemeinde jedoch wurden Schapers literarische Werke, sowie Essays und Reden rezipiert. 
Die drei Ortssprachen Estlands

Folgenden versucht, die Verwendung dieser Sprachen in der fiktionalen Welt für die Forschung der historischen Mehrsprachigkeit fruchtbar zu machen.

\section{Die drei Ortssprachen - Deutsch, Russisch, Estnisch}

Die estnische Gesellschaft ist historisch durch Mehrsprachigkeit gekennzeichnet. Bis zum Anfang des 13. Jahrhunderts wurden in den Gebieten des heutigen Estlands mündliche Varietäten des Estnischen verwendet (vgl. Hennoste 1997: 48). Die durch die Christianisierung im 13. Jahrhundert geprägten Machtverhältnisse führten zu einer Sprachhierarchie mit Latein an der Spitze, dem von den Kolonisten gesprochenen Niederdeutsch danach und den von der überwiegenden Mehrheit der Bevölkerung gesprochenen estnischen Varietäten an der niedrigsten Stelle. Ungefähr seit dem Ende des 14. Jahrhunderts begann Niederdeutsch Latein aus der Verwaltung herauszudrängen und obwohl Latein seine Position im kirchlichen und akademischen Leben länger hielt, nahm Niederdeutsch als Sprache der Oberschicht allmählich die erste Stelle in der Sprachhierarchie ein. Im 16. und 17. Jahrhundert wurde Niederdeutsch durch das Hochdeutsche ersetzt, aber dies betrifft vor allem die schriftliche Verwendung, im Mündlichen wurde Niederdeutsch teilweise bis zum 19. Jahrhundert benutzt (vgl. Lukas 2021: 16). Das hohe Prestige des Deutschen blieb auch während der schwedischen und russischen (in einigen Regionen des Landes auch dänischen und polnischen) Herrschaft erhalten, genauso wie der niedrigste Status des Estnischen. ${ }^{7}$ Ähnlich den anderen osteuropäischen Gebieten war der soziale Stand eines der definierenden Merkmale einer Nation - die höhere und untere Schicht sprachen unterschiedliche Sprachen (vgl. Hiio 2016: 11). Soziale Mobilität war kaum möglich, aber wenn diese doch vorkam, bedeutete es auch die Veränderung der Sprache und Nationalität innerhalb einer Generation (vgl. ebd. 18).

Obwohl im multiethnischen russischen Kaiserreich, wozu das Territorium des heutigen Estlands seit dem 18. Jahrhundert gehörte, sowohl die deutschals auch estnischsprachige Bevölkerungsgruppe zahlenmäßige Minderheiten waren, spielte die russische Sprache bis zur Russifizierung in den letzten Jahrzehnten des 19. Jahrhunderts in den estnischen Gebieten keine

\footnotetext{
Die baltische literarische Kultur besteht aus den lateinischen, niederdeutschen, deutschen, südestnischen, nordestnischen, lettischen, latgalischen, russischen, schwedischen, polnischen, griechischen, französischen, weniger aus den hebräischen, italienischen, spanischen, tschechischen, englischen und niederländischen Texten, die von ihren Autoren in schriftlich und mündlich öfters abwechselnd verwendet wurden (vgl. Lukas 2021: 18-19).
} 
JÄNES, SAAGPAKK

wesentliche Rolle und kann eher als eine Minderheitensprache berücksichtigt werden (vgl. Hennoste 1997: 53). Die Russifizierung beeinflusste alle Bevölkerungsgruppen. Der Einfluss und die Anzahl der russischsprachigen Gruppe vergrößerte sich, der deutschbaltische Teil der Bevölkerung, dessen privilegierter Stand zu zerfallen begann, musste sich langsam an die veränderten wirtschaftlichen und politischen Rahmenbedingungen anpassen (vgl. Woodworth, Tannberg 2009: 15). Auf den estnischen Teil der Bevölkerung hatte die Russifizierung eine ambivalente Wirkung (vgl. Raun 2009: 164-165). Seit der Mitte des 19. Jahrhunderts war es, wie vielerorts in Mittel- und Osteuropa, unter der estnischsprachigen Bevölkerung zu einem Erwachen des Nationalgefühls gekommen, woraus eine kulturell turbulente Entwicklung resultierte. Der mit der Russifizierung eingeleitete Versuch, die Machtverhältnisse neu aufzumischen, verstärkte die Fixierung auf die nationale Identität unter der estnischen Bevölkerung. Unter den Deutschbalten, die sich früher an Deutschland orientiert hatten, bildete sich als Folge des verstärkten nationalen Selbstbewusstseins der Esten, der langen Isolation von Deutschland und der beginnenden Russifizierung eine baltische geokulturelle Identität heraus (vgl. Lukas 2006: 40), die über die deutsche Identität zu dominieren begann.

Für das sprachliche Umfeld bedeuten die genannten Prozesse, dass die im Roman geschilderte estnische Gesellschaft am Anfang des 20. Jahrhunderts dreisprachig war - als Erstsprache sprach die Mehrheit der Bevölkerung Estnisch, die deutschbaltische Minderheit Deutsch, unter der russischen Minderheit sowie im öffentlichen Leben und in der Verwaltung war Russisch gängig. ${ }^{8}$ Die Grenzen waren natürlich nicht eindeutig und obwohl das Russische an der Spitze der offiziellen Sprachhierarchie lag, war die Sprachsituation in der Gesellschaft vielfältiger. Alle drei Ortssprachen waren je nach der Zugehörigkeit zu einer sprachlichen Gruppe an der ersten Stelle der persönlichen Sprachhierarchie (vgl. Hennoste 1997: 58). Obwohl ein Sprachwechsel nicht mehr eine Voraussetzung der sozialen Mobilität war und die Bestrebungen der Esten, als Deutsche oder Russen zu erscheinen, nun eher negativ beurteilt wurden, war die deutsch- oder russischsprachige Bildung nach wie vor hochgeschätzt. ${ }^{9}$

8 Laut der Volkszählung 1897 wurde als ,Muttersprache' 90\% Estnisch, 4\% Russisch, 3,6\% Deutsch, 1\% Lettisch, 0,6\% Schwedisch, 0,5\% Jiddisch, 0,3\% Polnisch, etc. gesprochen (vgl. Raag 2008: 113).

9 Noch in den 1920er Jahren, in der Republik Estland, überschritt die Zahl der Schüler in deutsch- und russischsprachigen Gymnasien die Zahl der Nicht-Esten in der Bevölkerung (vgl. Hiio 2016: 27). 
Während der Schreibzeit des Romans am Ende der 1930er Jahre hatte die Estnische Republik bereits zwei Jahrzehnte existiert. Als einzige Staatssprache fungierte Estnisch. Vor dem Zweiten Weltkrieg gehörte Estland zu den homogensten Gebieten Europas und die Minderheiten machten nicht mehr als rund 12 Prozent der Bevölkerung aus (Eesti Statistika 1881-2000). ${ }^{10}$ Die Verwendung der Minderheitensprachen war gesetzlich geregelt, wobei z. B. „die Menschen aus deutscher, russischer und schwedischer Nationalität“ in den staatlichen Behörden ihre Sprachen benutzen konnten und in den Gebieten, wo die Sprecher der Minderheitensprachen in Mehrheit waren, in der Verwaltung die Minderheitensprachen benutzt werden durften (Sprachgesetz 1934). Die zwei größeren Minderheiten waren fortdauernd die russischund deutschsprachigen Bevölkerungsgruppen mit rund 92000 und 16000 Menschen von insg. 1,1 Millionen Einwohnern Estlands (Rahvastiku koostis ja korteriolud 1935: 104). ${ }^{11}$ Die Sprachsituation war natürlich komplexer, z. B. waren die Deutschbalten finanziell und gesellschaftlich weiterhin einflussreich. Obwohl man also vor dem zweiten Weltkrieg noch von Estnisch, Russisch und Deutsch als drei Ortssprachen sprechen kann (vgl. Johansen 1963: 92), hatten die Funktionen und Hierarchien der Sprachen sich inzwischen verändert.

Zur Zeit des Übersetzens am Anfang des 20. Jahrhunderts genoss Estnisch wieder den Status der Staatssprache Estlands und alle anderen Sprachen wurden als Fremdsprachen bezeichnet (Sprachgesetz 1995). Während die russischsprachige Minderheit mehr als ein Viertel der Bevölkerung bildete und Russisch, obwohl ohne einen amtlichen Status, breit verwendet wurde, hörte die Funktion des Deutschen als Ortssprache bereits vor 60 Jahren, als die Deutschbalten im Rahmen der Umsiedlung ${ }^{12}$ (1939-1940) und der darauffolgenden Nachumsiedlung (1941) die estnischen Gebiete verließen, auf.

Wie bereits erwähnt, gab es in den Gebieten des heutigen Estlands neben den russisch- und deutschsprachigen auch andere sprachliche Minderheiten,

10 Auf dem Territorium des heutigen Estlands war die Anzahl der Minderheiten noch kleiner, $8 \%$.

11 Laut der Volkszählung 1934 wurden als ,Alltagssprache‘ (langue habituelle) Estnisch, Russich, Deutsch, Schwedisch, Lettisch, Jiddisch, Ingermanisch, Romani, Finnisch, Polnisch, Englisch, Tatarisch, Hebräisch, Französisch, Dänisch, Litauisch, Türkisch, Tschechisch, Italienisch, Niederländisch, etc. gesprochen (Rahvastiku koostis ja korteriolud 1935: 104-109).

12 Die Umsiedlung der in Estland und Lettland ansässigen Deutschbalten geschah, nachdem der Molotow-Ribbentrop-Pakt vom 23.08.1939 unterschrieben worden war. Die Deutschen aus dem Baltikum wurden in den darauf folgenden Monaten in die damals von den Deutschen bereits eroberten Gebiete im Westen Polens umgesiedelt (,Wartheland') (vgl. Pistohlkors 1994: 541). 
JÄNES, SAAGPAKK

wie die Schweden, aber z.B. auch Juden, Letten und Finnen. Diese waren jedoch entweder relativ klein oder nur in bestimmten Regionen ansässig. So waren zwar neben den drei Sprachen auch andere präsent, wurden aber im 20. Jahrhundert weniger oder nur in bestimmten Bereichen benutzt und kommen im Roman weniger oder gar nicht vor. Deswegen konzentriert sich der vorliegende Beitrag auf die sogenannten drei Ortssprachen.

\section{Die manifeste und latente Verwendung der drei Ortssprachen} im Roman und ihre Vermittlung in der Übersetzung ins Estnische

Während Mehrsprachigkeit in linguistischen Studien häufig „relativ unproblematisch" als das Auftreten der unterschiedlichen Idiome von Nationalsprachen, Dialekten, Soziolekten etc. in einem Text aufgefasst wird, kann die literarische Mehrsprachigkeit breiter verstanden werden (vgl. Dembeck, Parr 2017: 10). Sie äußert sich neben der manifesten (direkten) Mehrsprachigkeit auch in unterschiedlichen latenten (indirekten) Formen. In Der Henker bildet die manifeste Mehrsprachigkeit nur einen Teil der benutzten Verfahren, und die sprachliche Vielfalt wird meistens in unterschiedlichen latenten Formen, durch Hinweise auf die anderssprachige Figurenrede, durch die Erwähnung des Akzents, etc. dargestellt. So kommen im Roman neben den manifest anderssprachigen Passagen, die auch auf der Textebene in einer anderen Sprache stehen, auch latent anderssprachige Passagen vor, die auf Deutsch wiedergegeben sind. Bei der Übersetzung ins Estnische handelt es sich vor allem um die semantische Übersetzung, wo der Ausgangstext im Mittelpunkt steht und möglichst adäquat wiedergegeben wird. Im Vergleich zu den homophonen Übersetzungen ist in den semantischen Übersetzungen die textinterne Mehrsprachigkeit zwangsläufig weniger ausgeprägt (vgl. Bloemen, Sepp 2017: 235). So kann man auch bei der vorliegenden Übersetzung behaupten, dass nicht alle Mehrsprachigkeitselemente ins Estnische übermittelt sind. Tatsächlich verringert sich sowohl in der manifesten als auch auf der latenten Ebene die Mehrsprachigkeit, allerdings kommen auch weitere mehrsprachige Elemente dazu, die man sowohl mit dem Handlungsort an der Grenze Est- und Livlands als auch mit der Übersetzungszeit am Anfang des 21. Jahrhunderts begründen kann.

Neben dem Deutschen, Russischen und Estnischen werden die anderen im Roman vorkommenden Sprachen wie Französisch, Italienisch und Latein nur zusammenfassend betrachtet und werden zusammen mit dem Deutschen behandelt. Obwohl die Handlung meistens an der Sprachgrenze zwischen Estnisch und Lettisch stattfindet, wird Lettisch nicht analysiert, weil es im Roman kaum vorkommt. 
Die drei Ortssprachen Estlands

\subsection{Deutsch}

Der Roman ist auf der Textebene hauptsächlich auf Deutsch, die Handlungsebene ist aber mehrsprachig. Die anderssprachigen Passagen sind mit wenigen Ausnahmen auf Deutsch wiedergegeben. Dies betrifft sowohl die russisch- und estnischsprachigen Gespräche als auch die Gespräche, die vermutlich in der lokalen Varietät durchgeführt werden. Im Baltikum wurde, auch während der Handlungs- und Schreibzeit, eine Varietät gesprochen, die abhängig vom Kontext der Entstehung und Entwicklung des Begriffs als ,die deutsche Sprache im Baltikum', ,baltisches Deutsch', ,das Deutschbaltische;, ,Baltendeutsch“ oder ,Baltisch“ genannt wird. ${ }^{13}$ Diese Varietät unterschied sich vom Standarddeutschen durch die stärkere Präsenz des Niederdeutschen, einige Elemente der Aussprache, die Verwendung des aus den Kontaktsprachen entlehnten Vokabulars, etc.

Im Roman wird die lokale Varietät erwähnt („das baltische Deutsch“ [Schaper 1941: 77] $]^{14}$ ) und manche Baltizismen werden auch manifest verwendet. Es handelt sich vorwiegend um Bezeichnungen der Prozesse oder Agenten der Lokalgeschichte in der Handlungszeit. In vielen Fällen handelt es sich um Lehnübersetzungen aus dem Estnischen, wie „die grauen Barone“ (147), „Borstenrussen“ (526) und „Waldbrüder“ (305). Diese Wörter werden nicht übersetzt, wohl aber erläutert. Bei den Borstenrussen folgt in Klammern: „anders nannte er getreu der Predigt des berühmten Pastors Körber die Bauarbeiter gar nicht mehr“ und bei den Waldbrüdern wird ergänzt: „hießen sie beim Volke“. Ähnlich wird auch bei den „Kirchenherrn“ in Klammern ergänzt: „wie das Volk seine Pastoren nannte“ (352). Explizit wird nur einmal darauf hingewiesen, dass es sich um einen Baltizismus handelt - es wird ein wohlhabender estnischer Junge als „der Erbsohn des guten, großen Illustgesindes“ beschrieben und in einer Fußnote mitgeteilt, dass das Gesinde im Baltischen einen Hof oder ein bäuerliches Anwesen bedeutet (81).

In der Übersetzung sind die Lehnübersetzungen aus dem Estnischen zurück ins Estnische übersetzt. Die erwähnten Baltizismen sind in der Übersetzung „hallparunid“ (Schaper, übers. von Kaugver 2009: 111ü) ${ }^{15}$, „harjuskvenelased“ (397ü), „metsavennad“ (229ü) und „kirikhärra“ (264ü). Bei den letzten zwei wird auch in der Übersetzung ergänzt, dass diese Ausdrücke vom Volk benutzt werden, so dass die Distanziertheit zwischen den Deutschbalten

13 Mehr dazu siehe Bender 2009: 35-62.

14 Die Beispiele aus dem Roman stammen alle aus dieser Ausgabe, es werden daher im Weiteren nur die Seitenzahlen in Klammern angegeben.

15 Die Beispiele aus der Übersetzung stammen alle aus dieser Ausgabe, es werden daher im Weiteren nur die Seitenzahlen mit, ü' in Klammern angegeben. 
JÄNES, SAAGPAKK

und Esten weitergegeben wird, aber die exotisierende Wirkung der Ausdrücke verloren geht. Der einzige Baltizismus, zu dem im Roman gesagt wird, dass es sich um das Deutschbaltische handelt, steht in der Übersetzung auf Estnisch Gesinde ist als „talu“ übersetzt (62ü) und der Leser erfährt nicht, dass an dieser Stelle ein Baltizismus stand. Es gibt allerdings auch manche Baltizismen, bei denen es sich um Entlehnungen aus dem Russischen handelt und die auch in der Übersetzung die Funktion behalten, das lokale Kolorit weiterzugeben. Exemplarisch dafür steht „Bumagenwirtschaft“ (373) mit der Erklärung in der Fußnote „Bumaga (russisch): Papier“, wofür in der Übersetzung lediglich „bumaagamajandus“ (280ü) steht.

Auch bei den Ortsbezeichnungen kommt Mehrsprachigkeit vor, die mit dem jeweiligen kulturellen und politischen Kontext im Zusammenhang steht. Bei den größeren Orten sowie bei Kreisen und Städten, die hauptsächlich in den heutigen Gebieten Estlands liegen, werden im Roman die deutschen Namensformen verwendet. Wie in mehrsprachigen Gesellschaften üblich, waren auch in Estland parallel mehrere Formen im Gebrauch. Schaper hält sich durchweg an die deutschsprachigen und verzichtet auf die estnisch- bzw. russischsprachigen Formen. Dies betrifft nicht nur die Beschreibungen aus der deutschbaltischen Sicht und die Diskussionen auf Deutsch, sondern z. B. auch die Handlung, die sich auf die zaristische Armee bezieht. So kommen im Gespräch im latenten Russischen z. B. „Reval“ (19), „Wargel“ (19) und „Tidenküll“ (63) vor. Im Kontext der estnischen Diskussionen, Gedanken und Beschreibungen werden die größeren Orte eher selten erwähnt, wenn doch, dann werden ebenso deutsche Formen benutzt, z. B. hatte es Illusti Jüri „sogar zwei Semester lang in Dorpat mit dem Studieren versucht“ (81). In der Übersetzung stehen bei allen Ortsnamen durchwegs die estnischsprachigen Formen. So hatte auch der in Dorpat studierte Doktor von Riesbiter (25) in der Übersetzung in Tartu studiert (19ü) und der estnische Student Konstantin Sirg, dessen Sätze „in einem harten und scharfen Deutsch [kamen]“ studiert auch in Dorpat, in der Übersetzung in Tartu, obwohl die Stadt seit 1893 offiziell Jurjew (Jurjev) hieß. Die russischen Namensformen der im Gebiet Estland liegenden Orte kommen weder im Roman noch in der Übersetzung zur Erwähnung.

Während in der Handlungszeit die Parallelformen der Ortsnamen gängig waren, wurden während der Schreibzeit weitgehend estnische Namensformen benutzt. Nach dem Ersten Weltkrieg wurde das Thema der Verwendung von estnischen Namen im Deutschen und Russischen aktuell und besonders in den 1930er Jahren wandte man diesbezüglich auch offiziell Druck an. Im damaligen Deutschland herrschten wiederum nationalistische Stimmungen, ebenso waren die deutschsprachigen Namensformen dem potentiellen Leser bekannter 
als die estnischen Formen. Dem heutigen estnischen Leser könnten allerdings viele von den deutschsprachigen Formen unverständlich bleiben, so dass die Tatsache, dass die Deutschbalten in der Übersetzung im latenten deutschsprachigen Gespräch die estnischen Namensformen benutzen, zur Vereinfachung beiträgt. Somit ist die Verwendung der Ortsnamen ein Aspekt, bei dem sich die Mehrsprachigkeit in der Übersetzung eher vergrößert.

Ebenso stehen im Roman die Namen der Gutshöfe auf Deutsch und in der Übersetzung auf Estnisch, falls es einen estnischsprachigen Namen gibt. Z. B. die Gutshöfe in der Passage „[...] wie rundum, jenseits der ungeheuren Moore an der estländisch-livländischen Grenze, ein Gut nach dem anderen in Flammen aufging, Parkenthal, Wannamois, Jeddefer und Fickel“ (273) sind in der Übersetzung „Tolli, Vanamõisa, Jädivere ja Vigala“ (206ü). Während es im Roman sonst um reale Orte geht, ist der Gutshof von Ovelacker ein fiktiver Ort Drostenholm. Besonders auffällig ist diese Tatsache in der Übersetzung, weil Drostenholm wegen der fehlenden estnischen Namensform deutsch bleibt. Die in der Übersetzung addierte Mehrsprachigkeit unterstreicht die Distanziertheit zwischen der Hauptfigur und der Landbevölkerung, aber auch zwischen ihm und den anderen Deutschbalten, deren Gutshöfe estnische Namen tragen.

Neben solchen Beispielen, wo die Mehrsprachigkeit im Roman durch die Analyse der Übersetzung besonders ersichtlich wird, sind auch die Erwähnungen des Deutschen interessant. Die Fremdheit von Ovelacker wird oft durch die Sprache ausgedrückt. So trifft er sich im Quartier der russischen Offiziere mit dem alten Notar: „Ovelacker hatte es nur einen Augenblick des Zögerns gekostet, weil er sich klar machen mußte, daß der alte Herr ihn deutsch angesprochen hatte und daß er - deutsch geantwortet hatte." (132). Im ersten Teil des Romans findet die Handlung vorwiegend im latenten Russischen statt und über die Familie von Ovelacker wird gesagt, dass sie „verrußt“ (77) ist. So findet während des Romans der Übergang vom Russischen zum Deutschen statt, nur eben mit einem ,Zögern' dazwischen. In diesem Kontext ist besonders interessant, dass wenn es um die Beziehungen zwischen den Esten und Deutschbalten und die Funktionen der Sprachen geht, dieselbe ,verrußte Hauptfigur „bedingungsloser Gegner" der „Umvolkung“ (415) ist. ${ }^{16}$

Die deutschsprachigen Figuren des Romans benutzen auch westeuropäische ,Bildungssprachen', die ihre Zugehörigkeit zu einem bestimmten sozialen Kreis markieren. So wird in Begriffen oder in festen Wortverbindungen Latein bevorzugt - „status quo“ (451), „Pastor loci“ (28) etc., Französisch benutzt man z.B. für die Vermittlung der Emotionen - z.B. beim Träumen von der Abreise: „Aber in den Süden möchte er, wenn es hierzulande Herbst würde

16 Genauer dazu im Kapitel 4.3. 
JÄNES, SAAGPAKK

[...]. Alles käme darauf an, die Reihenfolge: rouge - noir, rouge - noir, rouge rouge, noir" (519). Zudem werden ,Bildungssprachen' benutzt, wenn es um Wertschätzungen geht, z. B. „Corpus delicti“ (385), „norme du corps“ (455), „honneur des Standes“ (724). Alle diese Ausdrücke sind sowohl im Roman als auch in der Übersetzung mit anderer Schrift hervorgehoben - im in gotischer Schrift gedruckten Roman sind sie in lateinischer Schrift, in der in lateinischer Schrift gedruckten Übersetzung sind sie kursiv.

\subsection{Russisch}

Russisch kommt im Roman in manifester Form relativ selten vor und wenn, dann vor allem bei den Personen- und Ortsnamen, bei der Verwendung von Patronymen oder bei Sprachmischungen, von denen „Bumagenwirtschaft“ $z u-$ sammen mit den Baltizismen schon betrachtet wurde. Auf die Tatsache, dass die Handlung im ersten Teil des Romans fast ausschließlich auf Russisch spielt, gibt es nur wenige manifeste sprachliche Hinweise, wie z. B. die Verwendung der Personennamen mit dem Patronym („Sie, Wladimir Karlowitsch, wandte er sich an Möller" ([30]). In der Übersetzung werden dieselben Formen der Mehrsprachigkeit wie im Roman verwendet und die wenigen Unterschiede kommen auf der manifesten Ebene vor. Die Transkription der russischen Namen unterscheidet sich vom Roman, so steht $\mathrm{z}$. B. der schon erwähnte Personenname „Wladimir Karlowitsch“ in der Übersetzung „Vladimir Karlovitš“ (23ü).

Auch latent wird der russischsprachigen Handlung nur wenig Aufmerksamkeit gewidmet, z. B. wenn die Sprachbeherrschung der Esten kommentiert wird: „riefen etliche im mühsamen Russisch“ (44), „Sie verstünden schlecht Russisch, namentlich die älteren“ (52) oder „In stockendem, holperigem Russisch“ (135). Neben den Kommentaren zur Sprachbeherrschung wird im Roman auch gesagt, dass während der Aussagen der Esten vor Gericht „einer der Schreiber als Dolmetsch einspringen mußte" (154). Diese Beispiele sind nicht nur aus der Perspektive der Handlung vielsagend, wo sie die schwache Position der Esten im Gerichtsprozess unterstreichen, die sich nicht richtig verteidigen können, sondern illustrieren auch die damalige gesellschaftliche Lage im Allgemeinen. Obwohl in der Russifizierungszeit das Russische als die offizielle Sprache eingeführt wurde, war die Bereitschaft und Fähigkeit, das Russische zu benutzen, in der Bevölkerung je nach Gruppe unterschiedlich.

Hierbei soll daran erinnert werden, dass auch die Deutschbalten mit der offiziellen Sprachhierarchie mit Russisch an der Spitze nicht zufrieden waren und das Deutsche aus ihrer Sicht weiterhin an der ersten Stelle lag. Im Roman steht allerdings ein Deutschbalte im Mittelpunkt, der es bevorzugt, Russisch zu sprechen: 
Die Ovelackers waren ,verrußt'. Er hatte mit allen Menschen russisch gesprochen, mit dem Pastor, mit dem Arzt, mit allen, so mühsam sie es auch radebrechten, der Arzt ausgenommen; er hatte nicht sein Deutsch sprechen wollen, das ihm, schon wenn er es nur sich selber vernehmbar vor sich hin sprach, ganz anders klang als das baltische Deutsch. (77)

Das Beispiel schildert die Akzeptanz der Russifizierung unter den Deutschbalten, ähnlich wie im Abschnitt: „[...] schon war die Sprache in ihren Häusern russisch geworden mit den Namen der Kinder und den Sitten“ (73). Während die Esten im Roman fast kein Russisch sprechen, sind die Russischkenntnisse der im Roman vorkommenden Deutschbalten mehr oder weniger vorhanden. Manifest stehen alle diese Passagen im Standarddeutschen und in der Übersetzung im Standardestnischen und es gibt keine manifesten Hinweise auf der Textebene auf die ,Mühsamkeit' der auf Russisch durchgeführten Gespräche.

Die damalige politische und kulturelle Situation wird aber durchaus auch mit manchen russischsprachigen Elementen illustriert. So gibt es einige Beispiele, die auf die Staatsverwaltung hinweisen wie „Urjadnik“, zu dem im Roman in der Fußnote die Übersetzung „Urjadnik (russisch): Landpolizist“ (321) steht. In der Übersetzung bleibt „Urjadnik“ (240ü) unübersetzt, da es auch im Estnischen gängig ist. Auffällig ist die Verwendung der Bezeichnung der Schwarzen Hundert, weil im Roman eine Mischung von deutschen und russischen Namen benutzt wird: „Schwarze Sotnja“ $(12,120)$. Bei diesem Beispiel kommt in der Übersetzung ebenso eine Verringerung der Mehrsprachigkeit vor, weil neben „Must Sotnja“ (91ü) auch „mustasajalised“ (10ü) verwendet wird. Ohne Erläuterung bleiben sowohl im Roman als auch in der Übersetzung einige weiteren Elemente des örtlichen Kolorits wie etwa im Deutschen „Kukuschka“ (442) $)^{17}$ und im Estnischen „Kukuška” (332ü). Während diese Elemente im deutschen Text auch als Deutschbaltisch kategorisiert werden könnten, erscheinen sie in der estnischen Übersetzung als Elemente des Russischen. Ähnlich den Namen sind auch alle anderen russischsprachigen Elemente sowohl im Roman als auch in der Übersetzung transliteriert, aber im Deutschen und Estnischen unterschiedlich transkribiert.

17 Кукушка-Russ. Kuckuck. 
JÄNES, SAAGPAKK

\subsection{Estnisch}

Das Estnische ist fast vom Anfang bis zum Ende des Romans präsent. Außer vielen Personen-, ${ }^{18}$ manchen Orts- und anderen Namen ${ }^{19}$ kommt Estnisch allerdings selten in manifesten Formen vor - die Esten sprechen wenig und sowohl ihr Gespräch als auch das Gespräch mit ihnen wird auf Deutsch weitergegeben. Wenn Estnisch manifest benutzt wird, trägt es eine symbolische Bedeutung, wie z. B. das Wort timukas, ${ }^{20}$ das im Roman neben dem Deutschen auch im Estnischen verwendet wird: „Er gewahrte Gestalten, die sich über eine Plattform hinauslehnten und irgend etwas schrien, was wie ,Timukas, Timukas!' klang [...]. [...] Sah ein ,Henker', wie sie da riefen, so aus?“ (315). In der Übersetzung steht das Wort „timukas“ (236ü) ebenso im Estnischen und verliert dadurch diese symbolische Bedeutung, die es im deutschen Text hat.

Neben den bereits beschriebenen deutschen Ortsnamen werden im Roman bei manchen Orten die estnischsprachigen Formen benutzt. Dies betrifft vor allem kleinere Orte, wobei auf das Fehlen ihrer deutschen Namen im Roman auch eingegangen wird: „Das wäre Köwerlaid, Krugholm im Deutschen. Diese Insel wäre groß genug für die deutsche Sprache“ (349). In der Übersetzung stehen ebenso sowohl der Name als auch dessen deutsche Übersetzung: „See olevat Kõverlaid, saksa keeles Krugholm.“ (261ü) Während die Namen der größeren und mit den Deutschbalten verbundenen Orte im Deutschen stehen, sind die estnischen Figuren von estnischsprachigen Ortsnamen umgeben. Auf dieselbe Weise wie Ovelacker mit den deutschsprachigen Namen assoziiert wird, ist die estnische Hauptfigur Koiri-Jaan mit dem estnischen Kontext, der auch durch die Namen reflektiert wird, verbunden. Exemplarisch dafür stehen „Er fuhr in den eisigen Wind, der vom Kibejöggi-Moor herüberkam, dem großen Morast um das Bett des ,bitteren Flusses““ (220) oder „Aber mit diesem Schmerz war es wie mit den Wassern des Salajöggi“21 (246). In beiden Fällen werden die estnischsprachigen, auf Emotionen basierenden Ortsnamen benutzt und deren Bedeutung dem deutschen Leser erklärt. Wie auch aus diesen Beispielen ersichtlich wird, benutzt Schaper bei Namen oft die alte bzw. deutsche Schreibweise, die im Estnischen sowohl während der Handlung wie auch während des Schreibens nicht mehr verwendet wurde. Die alte, am Deutschen orientierte Schreibweise für das Estnische war seit dem Ende des 17. Jahrhunderts bis zur Mitte des 19. Jahrhunderts in Gebrauch. Durch die

18 Z. B. Juhan (144), Kristjan (149), Tiiu (224), Mart (227).

19 Die überwiegende Mehrheit von den Orts- und anderen Namen stehen jedoch auf Deutsch.

20 Der Henker (Estn.).

${ }^{21}$ Auf Deutsch, Geheimnisfluss. 
Verwendung dieser Orthographie zeigt Schaper die Ausrichtung auf die deutsche Leserschaft. In der Übersetzung stehen die Namen auch auf der Textebene auf Estnisch - „Kibejõgi“ (166ü) und „Salajõgi“ (285ü) - somit wird die durch die Schreibweise sichtbare Sprachmischung nicht vermittelt ${ }^{22}$.

Auffällig ist auch die symbolische Namenswahl dieser Orte. Möglicherweise wird im Roman damit die historische koloniale Baltikumsperzeption der Deutschbalten weitergegeben, aber ebenso kann es durch die Schreibzeit des Romans erklärt werden. Wie gesagt, Schaper orientierte sich an deutschen Lesern, und eine seiner Absichten war es, „die urestnischen Elemente“ und „Eigenarten dieses Volkes“ zu präsentieren. ${ }^{23}$ Dieser Aspekt wird auch anhand mancher estnischsprachiger Personennamen ersichtlich, von denen der Hofname der estnischen Hauptfigur „Koiri“ besonders auffällig ist. Der Name ist klangähnlich mit dem estnischen Wort „koer“, welches Hund bedeutet. Darüber hinaus wird die Hauptfigur mehrere Male mit Tiermetaphern charakterisiert. Die Klangähnlichkeit wird im Text erwähnt: „Aber so etwas wie Koer oder Koeras oder Koiri hatte man draußen gesagt" (321). Da der Hintergrund des Namens im Roman nicht weitergegeben wird, bekommt der estnischsprachige Leser dies mit, der deutschsprachige aber nicht.

Dies ist nicht der einzige Fall, bei dem die estnischen Namen den Deutschbalten unklar bleiben, z.B. „Korras hieß er, glaube ich, oder so ähnlich.“ $\left(608^{24}\right)$ oder „Darauf stand ein belangloser Name, Konstantin Sirg oder so ähnlich“ (699). Während es sich bei den ersten zwei Beispielen um die Namen der estnischen Bauern handelt, die wegen der Mündlichkeit undeutlich bleiben, wird bei ,Sirg' auch der geschriebene Name eines estnischen Studenten auf der Visitenkarte von der deutschen Hauptfigur ähnlich abschätzig übergangen. Dies scheint ein weiteres Mittel zur Darstellung der gesellschaftlichen Beziehungen und der (Sprach-)Hierarchien zu sein.

22 Ähnliche Sprachmischung kommt auch bei manchen Personen- bzw. Hofnamen vor, wie z. B. „Leppiko“ (144) und „Pöldsche [Sippe]“ (469), die in der Übersetzung ,korri-

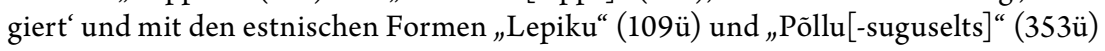
ersetzt sind.

23 Schapers Interview mit Vokk: - „Interessiert ein solcher Roman den durchschnittlichen deutschen Leser, der kaum glaubt, dass Estland keine Provinz Deutschlands ist", stelle ich dem Schriftsteller die Frage.“ - „Die Deutschen begehren Estland keinesfalls als Provinz, das ist ganz sicher. Und die deutsche Leserschaft ist an jedem Buch interessiert, wenn es denn tatsächlich urestnische Elemente präsentiert; wenn darin das Leben, die Kämpfe und Eigenarten dieses Volkes widergespiegelt sind.“ (1939: 10, Übersetzung M.J.)

24 ,Korras' bedeutet in Ordnung, hier hat der Name also eine eher positive Konnotation, wie auch ,Sirg', was an , sirge ${ }^{\prime}$ - gerade - angelehnt ist. 
JÄNES, SAAGPAKK

Neben den betrachteten manifesten Beispielen tritt Estnisch im Roman auch in latenten Mehrsprachigkeitsformen auf. Manchmal wird dabei explizit darauf hingewiesen, dass es sich um das Estnische handelt, manchmal nicht. Dies betrifft auch die Entlehnungen, die zur Illustration der estnischen Figuren eingesetzt werden: „Ein Kaiser brauchte nur einen starken Kopf, hatte Thomasson mit einer aus dem Estnischen geliehenen Wendung erwidert“ (310) und „mit dem Kolomats, dem estnischen Kinderschreck“ (378). Hier wird im Text gesagt, dass es sich um das Estnische handelt. Andere kulturelle Übernahmen bleiben aber verborgen. So fragt sich Olli, die estnische Magd von Koiri: „Zogen die Schwäne, kam jetzt der Schnee?“ (228) oder ein estnischer Jungknecht pfeift „Wie weit es noch bis Ösel ist ...“(507). Beim ersten Beispiel handelt es sich um eine Modifizierung einer estnischen Redewendung „Luiged lähevad, lumi taga." (172ü) und beim zweiten um eine Übersetzung einer Zeile des estnischen volkstümlichen Liedes „Kui kaugel on veel Saaremaa...“ (382ü). Dem Leser wird nicht gesagt, dass es sich um das Estnische handelt und der Hintergrund dieser Textstellen wird nicht erläutert. Ebenso wird im Roman die im Estnischen heutzutage weit verbreitete Äußerung „der dörptsche Geist“ (654) verwendet. Es handelt sich um eine Übersetzung des Ausdrucks „Tartu vaim“ (494ü), der in den 1930er Jahren in Bezug auf die damalige politische Situation in Estland zur Verwendung kam (Haug 2004: 90). Solche strukturellen Sprachmischungen fungieren im Roman und in der Übersetzung unterschiedlich, da sie im deutschen kulturellen Kontext unverständlich bleiben, im estnischen Kontext aber allgemein bekannt sind.

Estnisch bleibt in manchen latenten Passagen nicht nur unerwähnt, sondern auch unübersetzt. Vor allem hängt es damit zusammen, dass die Handlung aus der Perspektive Ovelackers beschrieben wird und für ihn die estnischsprachigen Gespräche unverständlich sind, wie z. B. im Abschnitt: „Eintönig, als läse sie vor, kam Wort um Wort mit der gleichen Betonung, ein Geriesel von Worten, die einen frommen Sinn haben mochten. Ihm ging die Bedeutung nicht auf.“ (744). Einen ähnlichen symbolischen Wert trägt Estnisch auch an anderen Stellen, wobei im Unterschied zu diesem Beispiel meistens nicht das Gespräch der Esten, sondern mit den Esten dargestellt wird. Meist spricht der Verwalter Estnisch, der als Vermittler zwischen der deutschsprachigen Herrschaft und estnischsprachigen Landbevölkerung agiert: „Und als nun der Verwalter an seiner Linken zu sprechen begann - in einer Sprache, die er nicht verstand [...]. Er sah den Platz und die Menschen [...], halb wie ein Gast und halb wie ein Gründer." (331). Während es in diesen Beispielen nicht wichtig ist, was gesprochen wird, sondern dass das Gespräch in einer anderen Sprache stattfindet, gibt es auch entgegengesetzte Beispiele, wie „[Der Verwalter] herrschte die Leute an: Was das heißen sollte, ob sie sich nicht packen wollen! [...]“ (314). Vermutlich handelt es sich auch hierbei um Estnisch, aber gesagt 
wird es nicht. Ebenso gibt es Passagen, wo auch die handelnden Figuren nicht sagen können, ob es sich um Deutsch oder um Estnisch handelte, wie z. B. „in welcher Sprache, wüßte sie gar nicht mehr“ (572).

Die Sprachfrage ist im Roman auch als thematisches Element wichtig. Es wird häufig über die Stellung und Funktion der drei Ortssprachen diskutiert. Dazu, dass die Sprache die Zugehörigkeit zu einer Gruppe bestimmt, gibt es im Roman zahlreiche implizite wie explizite Beispiele:

Weil sie sich nicht als Herren unter Knechten fühlten, sondern als Bürger unter Bürgern, waren die meisten der Deutschen, die erst in jüngster Zeit aus dem Reich zugezogen, den Alteingesessenen verächtlich geworden. Sie taten, was denen als schwere Verfehlung wider das koloniale Gesetz galt: sie heirateten ins Volk der Letten und Esten. Lettisch oder Estnisch wurde oft genug ihre Umgangssprache, ihre Kinder fühlten sich, kaum daß sie wußten, was sie fühlten, als Letten und Esten und verleugneten ihre Herkunft, wenn sie nicht sogar das Deutsche zu hassen begannen. Wie sprach der Verwalter mit ihm? Estnisch, so unvollkommen der Westfale das beherrschte. [...] Und es wäre besser so, hatte der Verwalter beharrt, wie lebhaft auch sein Gutsherr ihm widersprach. Eine Sprache für die Leute und eine andere, die die Leute am besten gar nicht verstünden, für die Herrschaft. Mit dem Förster estnisch zu sprechen, darauf wäre er natürlich nicht verfallen, weil dieser Reichsdeutscher war. Der gehörte zu den Herrschaften, war Reserveoffizier, besaß Manieren. Mit den einfachen Leuten die Sprache der Herrschaft sprechen zu müssen, war peinlich. Sie hatten ja nicht die Umgangsformen von Herrschaften und konnten das Ansehen des Deutschen nur vermindern. [...] Er, Ovelacker, hatte sich als bedingungsloser Gegner dieser Umvolkung bekannt, aber was half es, [...]. (404-405)

Solche Ausführungen verdeutlichen den soziokulturellen Hintergrund der Sprachverwendung und zeichnen die Statusunterschiede zwischen Deutsch und Estnisch nach. Die strikte Teilung und unterschiedliche Funktionen der Sprachen waren für das Baltikum charakteristisch und die Sprache definierte die gesellschaftliche Position und Haltung. Daneben aber lenkt der Abschnitt die Aufmerksamkeit auf die Risse, die in dieser festen Sprachhierarchie vorkamen und zeigt, dass die Bewegung zwischen den Gruppen unter einigen Bedingungen doch möglich war. Dies hilft auch, den symbolischen Wert des Romanendes besser zu kontextualisieren, wenn der Deutschbalte Ovelacker auf Estnisch zu sprechen anfängt: „Ich werde schreiben, daß der... der Junge kommt! fügte er in seinem mühsamen Estnisch hinzu, ohne auch nur im mindesten zu zögern, ohne daß sein Stolz sich dawider empörte." (749). Dieser latent dargestellte Sprachwechsel unterstreicht den symbolischen Akt der Versöhnung. 
JÄNES, SAAGPAKK

\title{
5 Zusammenfassung
}

Die gesellschaftliche Mehrsprachigkeit äußert sich in Der Henker sowohl in manifesten als auch in latenten Formen und es werden besonders die drei Ortssprachen verwendet. Die manifeste Mehrsprachigkeit beschränkt sich auf die vielen Personen-, Orts-, Zeitungs- und anderen Namen sowie auf manche Textstellen zur Markierung lokaler Elemente. Der überwiegende Teil der Mehrsprachigkeit wird durch latente Formen weitergegeben. Die Übersetzung geht von den semantischen Grundlagen aus, aber die damit verbundene Voraussetzung, dass die Mehrsprachigkeit sich beim Übersetzen verringert, gilt nur teilweise. Da in der Übersetzung die Vermittlung aus einer Ortssprache in die andere Ortssprache stattfindet, gibt es auch Stellen, wo sich die Mehrsprachigkeit in der Übersetzung vergrößert.

Schaper sah als Zielgruppe seines Romans die deutschsprachige Leserschaft und die Tatsache, dass es kaum manifeste Erscheinungen der Mehrsprachigkeit gibt, kann mit der Orientiertheit an einsprachige Leser sowie mit der Zeit des Verfassens im Zusammenhang stehen. So gibt es nur wenige sprachliche Hinweise darauf, dass die Handlung im ersten Teil des Romans hauptsächlich im Russischen stattfindet, ebenso ist die deutschbaltische Varietät, die im Baltikum während der Handlung gesprochen wurde, nur durch wenige Ausdrücke vertreten. Obwohl es auch Diskussionen über das Russische gibt und Deutschbaltisch erwähnt wird, räumt der Roman dem Estnischen eine besondere Stellung ein. Die Funktion des Estnischen besteht darin, den kulturellen Kontext und die gesellschaftlichen Beziehungen, aber auch den gesellschaftlichen Wandel zu illustrieren. Der Sprachwechsel des Protagonisten am Ende des Romans vom hierarchisch höherstehenden Deutschen ins Estnische kann als eine Wertung der historischen Prozesse gelesen werden. Somit wird die Mehrsprachigkeit als ein Aspekt der Standeshierarchie abgebildet und zugleich hinterfragt.

\author{
Marin Jänes \\ marin@utkk.ee \\ Academy of Sciences \\ ESTONIA \\ Maris Saagpakk \\ saagpakk@tlu.ee \\ Tallinn University \\ ESTONIA
}

Tallinn University / Under and Tuglas Literature Centre of the Estonian 
Die drei Ortssprachen Estlands

\section{Literatur}

Adson, A. 1958. E. Schaper, Sie mähten gewaffnet die Saaten, 499 S, Jakob Hegneri kirjastus Kölnis, 1956. - Mana, 2, 57-59.

Arens, O. 2009. Edzard Schaper. Timukas. - Akadeemia, 3, 603-610.

Bender, R. 2009. Oskar Masing und die Geschichte des Deutschbaltischen Wörterbuchs. Tartu: Tartu Ülikooli Kirjastus.

Bloemen, H., Sepp, A. 2017. Semantische Übersetzung. - T. Dembeck, R. Parr, Hrsg., Literatur und Mehrsprachigkeit: Ein Handbuch. Tübingen: Narr Francke Attempto Verlag, 235-248.

Dembeck, T., Parr, R. 2017. Mehrsprachige Literatur. Zur Einleitung. - T. Dembeck, R. Parr, Hrsg., Literatur und Mehrsprachigkeit: Ein Handbuch. Tübingen: Narr Francke Attempto Verlag, 9-14.

Ex-. 1940. Sakslase romaan 1905. aasta timukast. - Rahvaleht, 8.05, 107, 10.

Gierlich, E. 2014. Zum Geleit. - C. Sauerland, E. Gierlich, Hrsg., Edzard Schapers Blick auf die Totalitarismen seiner Zeit, Literarische Landschaften, Bd. 15. Berlin: Duncker \& Humblot, [5].

Haug, T. 2004. Troojamäe taotlus. Tallinn: Eesti Keele Sihtasutus.

Hennoste, T. 1997. Eesti keele sotsioperioodid. Üldpilt. - M. Erelt, M. Sedrik, E. Uuspõld, Hrsg., Pühendusteos Huno Rätsepale, Tartu Ülikooli eesti keele õppetooli toimetised 7. Tartu: Tartu Ülikool, 45-66.

Hiio, T. 2016. Meie võõrad ja meie omad. Saksastumisest, ülikooliharidusest ja karjäärist Heinrich Rosenthalist Feliks Urbaninini. - L. Leppik, Hrsg., Tartu Ülikooli ajaloo küsimusi. Tartu: Tartu Ülikooli muuseum, 9-36.

Iliste, I. 1998. 1905 im historisch-literarischen Kontext. Vergleichende Betrachtungen zu Edzard Schaper. - K. Lepa, C. Sommerhage, Hrsg., Triangulum. Sonderheft: Edzard Schaper. Tartu: TÜ Kirjastus, 129-147.

Johansen, P. 1963. Nationale Vorurteile und Minderwertigkeitsgefühl als sozialer Faktor im mittelalterlichen Livland. - Historisches Seminar der Universität Hamburg, Hrsg. Alteuropa und die moderne Gesellschaft. Festschrift für Otto Brunner. Göttingen: Vandenhoeck \& Ruprecht.

Lepik, V. 1959. Edzard Schaper ja tema looming. - Mana, 3, 170-184.

Lukas, L. 1999. Piiririik - Eesti kujund Edzard Schaperi loomingus. - Looming, 7, 1061-1072.

Lukas, L. 2006. Baltisaksa kirjandusväli 1890-1918. Tartu-Tallinn: Underi ja Tuglase Kirjanduskeskus, Tartu Ülikooli kirjanduse ja rahvaluule osakond.

Lukas, L. 2009. Järelsõna. Edzard Schaperi lepitusromaan. - E. Schaper, Timukas. Liivimaa klassika. Tallinn: Eesti Päevaleht, 567-575.

Lukas, L. (Hrsg.) (2021). Balti kirjakultuuri ajalugu I. Keskused ja kandjad. Tartu: Tartu Ülikooli Kirjastus.

Neuschäffer, H. 1982. Der historische Roman Der Henker von Edzard Schaper und der geschichtliche Hintergrund von 1905. - A. Ezergailis, G. von Pistohlkors, Hrsg., Die baltischen Provinzen Rußlands zwischen den Revolutionen von 1905 und 1917. Köln-Wien: Böhlau, 245-256. 
JÄNES, SAAGPAKK

Otto-Sprunck, A. 1994. Eestimaal tundmatu: Edzard Schaper, südame-eestlane. Akadeemia, 3, 503-508.

Pistohlkors, G. v. 1994. Deutsche Geschichte im Osten Europas. Baltische Länder. Berlin: Siedler.

Raag, R. 2008. Talurahva keelest riigikeeleks. Tartu: Atlex.

Rahvastiku koostis ja korteriolud. 1935. 1. III 1934 rahvaloenduse andmed, Vol. II. Tallinn: Riigi Statistika Keskbüroo.

Raun, T. U. 2009. Venestamine Eestis 19. sajandi teisel poolel ja 20. sajandi algul. T.-A. Tannberg, B. Woodworth, Hrsg., Vene impeerium ja Baltikum: venestus, rahvuslus ja moderniseerimine 19. sajandi teisel poolel ja 20. sajandi alguses, Eesti Ajalooarhiivi toimetised, 16 (23). Tartu: Eesti Ajalooarhiiv, 113-165.

Rosenwald, A. L. 2008. Multilingual America. Language and the Making of American Literature. Cambridge: Cambridge University Press.

Rutten, G., Salmons, J., Vandenbussche, W., Vosters, R. 2017. Unraveling multilingualism in times past. The interplay of language contact, language use and language planning. - Sociolinguistica, 31 (1), 9-20. https://doi.org/10.1515/soci-2017-0002

Saagpakk, M. 2014. „Der Henker“ von Edzard Schaper aus postkolonialer Sicht. - K. Sauerland, E. Gierlich, Hrsg., Edzard Schapers Blick auf die Totalitarismen seiner Zeit. Berlin: Duncker \& Humblot, 47-63.

Schaper, E. 1941. Der Henker. Leipzig: Insel.

Schaper, E. 2009. Timukas (übers. v. Katrin Kaugver). Tallinn: Eesti Päevalehe AS.

Sprachgesetz. Riigi Teataja 1934, 93.

Sprachgesetz. Riigi Teataja 1995, 23, 334.

Statistikaamet. Eesti Statistika. Rahvaarv 1881-2000.

Tannberg, T.-A., Woodworth, B. 2009. „Imperiaalne pööre“ paljurahvuselise Vene impeeriumi ajaloo uurimisel. Saateks. - T.-A. Tannberg, B. Woodworth, Hrsg., Vene impeerium ja Baltikum: venestus, rahvuslus ja moderniseerimine 19. sajandi teisel poolel ja 20. sajandi alguses, Eesti Ajalooarhiivi toimetised, 16 (23). Tartu: Eesti Ajalooarhiiv, 5-15.

Undusk, J. 1999. Rääkimise reetlik puhtus. Edzard Schaper oma aja kõnesituatsioonis. - Looming, 7, 1079-1087.

Vokk, E. J. 1939. Saksa sulg kirjeldab eesti elu. - Rahvaleht, 18.01, 15, 10.

Wilpert, G. von. 2005. Deutschbaltische Literaturgeschichte. München: Beck. 